\title{
Novel Software for the Assignment of Peaks from Tandem Mass Spectrometry Spectra of Synthetic Polymers
}

\author{
Konstantinos Thalassinos, ${ }^{\mathrm{a}}$ Anthony T. Jackson, ${ }^{\mathrm{b} *}$ Jonathan P. Williams, ${ }^{\mathrm{a}}$ \\ Gillian R. Hilton, ${ }^{a}$ Susan E. Slade, ${ }^{a}$ and James H. Scrivens ${ }^{a}$ \\ ${ }^{a}$ Department of Biological Sciences, University of Warwick, Gibbet Hill Road, Coventry, UK \\ ${ }^{\mathrm{b}}$ Measurement Science Group, ICI plc, Wilton Centre, Redcar, Cleveland, UK
}

Novel software has been developed to aid the interpretation of tandem mass spectrometry (MS/MS) data from synthetic polymers. The software is particularly focused toward aiding the end-group determination of these materials by significantly speeding up the interpretation process. This allows information on the initiator and/or chain transfer agents, used to generate the polymer, and the mechanism of termination to be inferred from the data much more rapidly. The software allows the validity of hypothesized structures to be rapidly tested by automatically annotating the data file using previously proposed fragmentation rules for synthetic polymers. Low-energy collision-induced dissociation (CID) data from methacrylate, styrene, and polyether oligomers are used as example data for the software. Exact-mass CID information was used to aid the understanding of the dissociation mechanism of the polymers. The software can use exact-mass data to provide more confidence in the results. The MS/MS results indicate that the fragmentation pathways are those previously proposed for these polymers. (J Am Soc Mass Spectrom 2007, 18, 1324-1331) (c) 2007 American Society for Mass Spectrometry

S oftware and databases are an inherent tool for the identification of peptides and proteins from tandem mass spectrometry (MS/MS) data [1-6]. These MS/MS data are typically the result of lowenergy collision-induced dissociation (CID) experiments. Until now there has been no development of software that might be used to aid the interpretation of MS/MS data from synthetic polymers. The development and application of novel software for the interpretation of MS/MS data from a range of synthetic polymers-including acrylics, polyethers, and styrenes-are described below. These MS/MS data are primarily used to aid the end-group determination of synthetic polymer systems, often in conjunction with other techniques such as matrix-assisted laser desorption/ionizationtime-of-flight (MALDI-TOF) mass spectrometry and nuclear magnetic resonance (NMR) spectroscopy. There is, indeed, a powerful synergy between these three techniques for end-group determination.

The software uses fragmentation schemes that have been proposed by Jackson and coworkers [7-17] and others, such as Lattimer and Wesdemiotis et al. [18-21]. The structures of end groups from acrylic and styrene polymers may often be inferred from the masses

Address reprint requests to Dr. A. T. Jackson, Measurement Science Group, ICI plc, Room D115, Wilton Centre, Redcar, Cleveland, TS10 4RF, UK. E-mail: tonyjackson@ici.com

* Current address: Materials Characterization Section, ICI Paints, Wexham Road, Slough, Berkshire, SL2 5DS. (and/or exact masses) of two series of intense peaks seen in the low mass-to-charge ratio $(\mathrm{m} / \mathrm{z})$ region of the CID spectra [7-14, 16]. It has been noted, however, that the higher $\mathrm{m} / \mathrm{z}$ peaks in the low-energy CID spectra are more useful in aiding understanding of the identity of end-group structures of polyethers [such as poly(ethylene glycol) (PEG) and poly(propylene glycol) (PPG)] $[15,17]$. Software-aided interpretation tools are essential if microstructural studies of synthetic polymers are to be more widely used.

The software can be used with exact-mass-MS/MS experiments to give further confidence in the assignment of end-group structures. Example data for poly(methyl methacrylate) (PMMA), poly(butyl methacrylate) (PBMA), PEG, PPG, poly(styrene), and $\operatorname{poly}(\alpha-$ methyl styrene) are used to display how exact-massMS/MS data may imported and interpreted with the software.

\section{Experimental \\ Software Development}

The software was written in Java v1.4.2 (Sun Microsystems, Santa Clara, CA, USA). The graphical user interface of the software is used to enter the information required to calculate the polymer fragments. These are the polymer class, repeat unit, initiating and terminating end groups, number of repeats, and cation. Both 
types of chemical structure representation are acceptable such as "CH3CH2" or "C2H5." The MS/MS data to be annotated are read from an ASCII text file containing mass intensity information. Given a user-selected mass accuracy error (in Daltons), fragments are computed by the software and are matched to experimentally observed peaks. Each matched peak is displayed with a color corresponding to each fragment type and annotated with the fragment information. Matched peaks are also displayed in a table. The user can interact with the table and edit the peak annotation, add annotation to peaks not matched by the software, or delete the annotation from incorrectly assigned peaks.

\section{Tandem Mass Spectrometry}

Electrospray ionization-tandem mass spectrometry (ESI-MS/MS) experiments were performed in a hybrid quadrupole time-of-flight mass spectrometer (Q-TOF Ultima, Waters MS Technologies, Manchester, UK). The instrument was equipped with a standard electrospray ion source and operated at a source temperature of $110^{\circ} \mathrm{C}$. Sample solutions were introduced into the source region of the instrument by a direct infusion ESI, operated in positive mode of ionization, with a capillary voltage of $3.5 \mathrm{kV}$. The cone voltage was optimized between 50 and $200 \mathrm{~V}$ for ESI-MS/MS experiments and the collision energy was ramped between 20 and $100 \mathrm{eV}$ during the acquisition. ESI-MS/MS was carried out using argon as the collision gas. The TOF mass analyzer was tuned in $\mathrm{V}$-optic mode for an operating resolution of 10,000 (full width at half-maximum). Mass spectra were acquired at an acquisition rate of one spectrum/s with an interscan delay of $100 \mathrm{~ms}$. Data acquisition and processing were carried out using MassLynx ${ }^{\mathrm{TM}}$ (V4.0) software (Waters Corp., Milford, MA).

\section{Sample Preparation}

PEG 400 and PPG 425 were obtained from SigmaAldrich (Gillingham, UK). PMMA 2400 and poly(styrene) 2050 were obtained from Polymer Laboratories (Church Stretton, UK). Poly( $\alpha$-methyl styrene) 1580 and PBMA 2800 were from Polymer Standards Service (Mainz, Germany). Lithium chloride, silver trifluoroacetate, methanol, and tetrahydrofuran (THF) were obtained ${ }^{\circ}$ from $^{\circ}$ Sigma-Aldrich. ${ }^{\circ}$ Poly $(\text { styrene })^{\circ}$ and ${ }^{\circ} \operatorname{poly}(\alpha-$ methyl styrene) were dissolved in THF to a concentration of $200 \mathrm{ng} / \mu \mathrm{L}$. Silver trifluoroacetate was dissolved in THF to a concentration of $5 \mathrm{mg} / \mathrm{mL}$. ESI-MS/MS analysis of poly(styrene) and poly $(\alpha-$ methyl styrene) were performed by infusing a mixture of sample and silver trifluoroacetate (to promote cationization) at a ratio of 1:1. PEG, PPG, PMMA, and PBMA were dissolved in methanol to a concentration of 100 $\mathrm{ng} / \mu \mathrm{L}$. Lithium chloride was dissolved in $50 \%$ aqueous methanol to a concentration of $2 \mathrm{mg} / \mathrm{mL}$. ESI-MS/MS analysis of PEG, PPG, PMMA, and PBMA was performed by infusing a mixture of sample and lithium chloride at a ratio of 1:1. All samples were infused into the source region of the mass spectrometer at a rate of $10 \mu \mathrm{L} / \mathrm{min}$.

\section{Results and Discussion}

Tandem mass spectrometry (MS/MS) data from a number of standard synthetic polymers are used to emphasize how the software may be used to aid interpretation and infer information on their end-group structure by significantly reducing the time taken for this process. The proposed fragmentation mechanisms for the polymers $^{\circ}$ were $^{\circ}$ previously $^{\circ} \operatorname{described}^{\circ}\left[7,{ }^{\circ} 8,{ }^{\circ} 10,{ }^{\circ} 12,{ }^{\circ} 19,{ }^{\circ} 20\right]$, with these used by the software to annotate the respective peaks in the MS/MS spectra. The spectra are imported into the software as text files, with a capability for analysis of both exact-mass and nominal mass MS/MS data from a range of polymer classes.

MS/MS has been used to aid the end identification of anumber ${ }^{\circ}$ of $^{\circ}$ methacrylate ${ }^{\circ}$ polymers $[7-9,91,913,914,916]$, of which PMMA is the most common example. This polymer is used in a range of applications, partly as a consequence of its optical clarity. End-group functionalization of PMMA is important in a number of applications of the polymer and understanding the nature of the initiating and terminating groups can help polymer chemists in deciphering the route of manufacture. An example of how the software can be used to aid interpretation of MS/MS spectra of PMMA (1) is shown in ${ }^{\circ}$ Figure ${ }^{\circ} 1$.

The ESI-MS/MS spectrum from the lithiated octadecamer (18-mer) of PMMA was imported into the software as a text file and the suggested repeat unit, initiating (alpha, $\alpha$ ), and terminating (omega, $\omega$ ) end groups were input as empirical formulae $\left(\mathrm{C}_{5} \mathrm{H}_{8} \mathrm{O}_{2}\right.$, $\mathrm{C}_{5} \mathrm{H}_{9} \mathrm{O}_{2}$, and $\mathrm{H}$ in the "Main Unit," "Alpha," and "Omega" input boxes, respectively). The class of polymer ("Acrylates") was selected, the cation used to ionize the oligomer ( $\mathrm{Li}$ in "Cation"), the number of repeat units (17 in "Repeats"), and the mass accuracy (0.2 Da in "Error (Da)," as the data were acquired without the precursor ion, or an external calibrant, used as lock mass were both entered and the "Annotate" button was selected. The resulting annotated spectrum is ${ }^{\circ}$ displayed ${ }^{\circ}{ }^{\circ}{ }^{\circ}$ Figure 1 , ${ }^{\circ}$ along ${ }^{\circ}$ with $^{\circ}{ }^{\circ}$ table $^{\circ}$ of ${ }^{\circ}$ proposed fragment ions. The latter details the calculated $\mathrm{m} / \mathrm{z}$ of the fragment matched ("Theoretical Mass"), the fragment annotation ("Description"), the experimental $\mathrm{m} / \mathrm{z}$ ("Observed Mass"), the difference between theoretical and experimental $m / z$ in Daltons ["Error (Da)"], and the relative intensity of the matched peak ("Intensity"). The color used to highlight each fragment ion is also shown in the "Highlight Color" column of this table, with a series $^{\circ}$ of $^{\circ}$ peaks $^{\circ}$ (e.g., ${ }^{\circ}$ from $^{\circ}$ the $^{\circ} \mathrm{A}^{\circ}$ series $^{\circ}$ in $^{\circ}$ Figure $^{\circ} 1$ ) displayed in the same color. The annotation and highlighting of each peak can be temporarily removed using the "Display" column of the table (clicking on the checkbox in that column for the relevant peak) or 


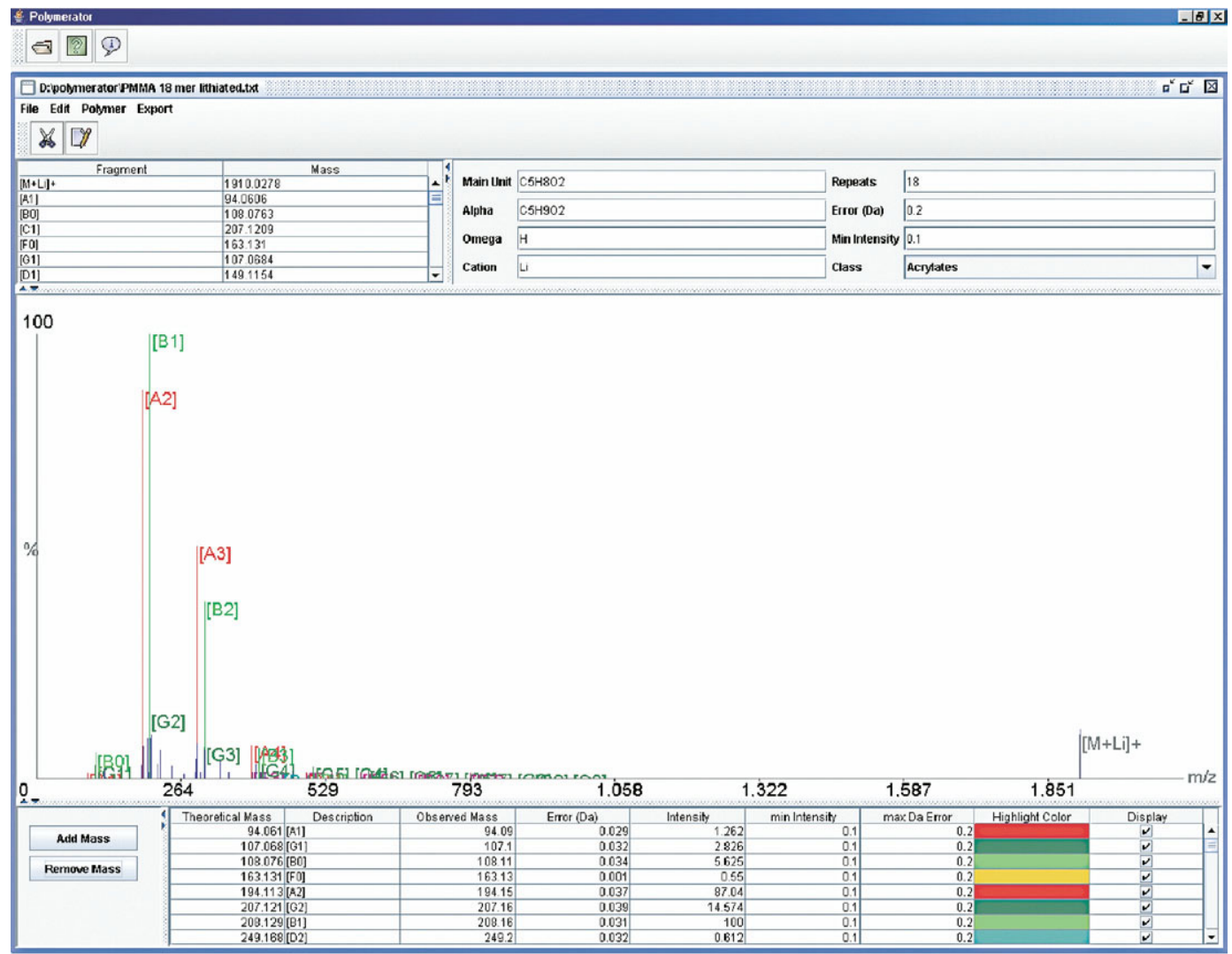

Figure 1. Screenshot from software of annotated ESI-MS/MS spectrum from the lithiated octadecamer of PMMA. Details of annotated fragment ions are displayed by the software in the table below the spectrum. Predicted fragment ions are also detailed above (left) of the spectrum.<smiles>COC(=O)C1CC(C)C1CCC(C)(C)C(=O)OC</smiles>

1 custom annotation and/or coloring can be added as required. This means that peaks originating from a novel fragmentation pathway, for example, can be annotated if required. This could be useful when annotating MS/MS spectra of PMMA that contain haloge-

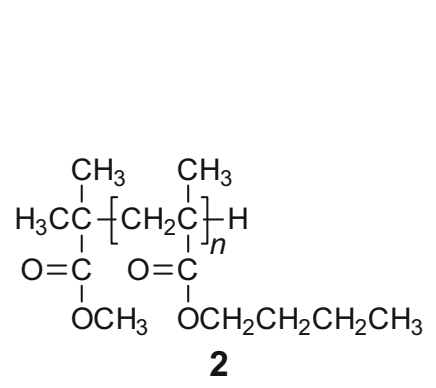

2

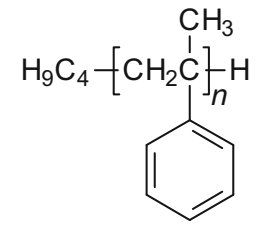

4

$$
\mathrm{HO}-\left[\mathrm{CH}_{2} \mathrm{CH}_{2} \mathrm{O}\right]_{n} \mathrm{H}
$$

5 


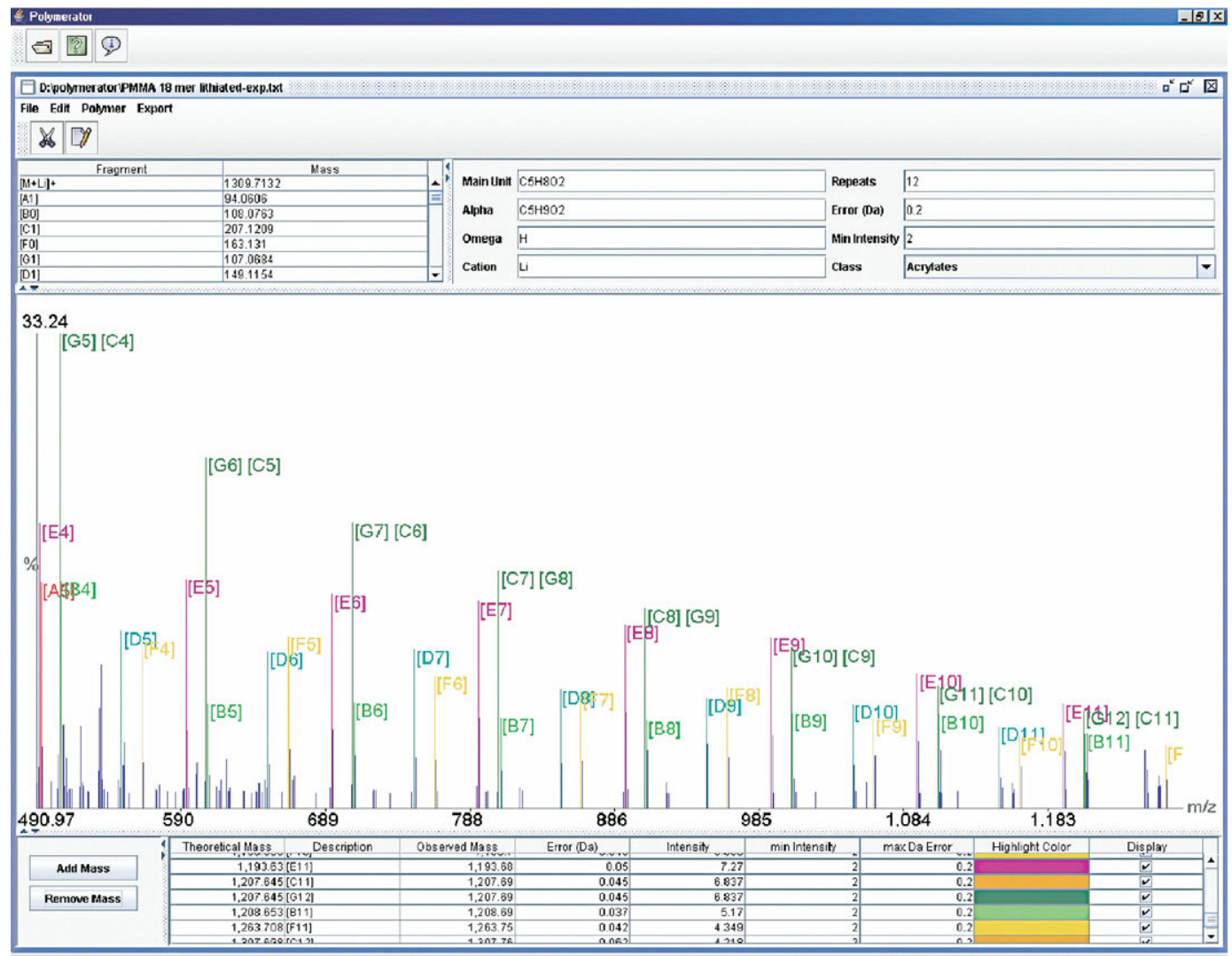

Figure 2. Screenshot from software of annotated expansion $(m / z \cong 490-1250)$ of ESI-MS/MS spectrum from the lithiated octadecamer of PMMA. Details of annotated fragment ions are displayed by the software in the table below the spectrum. Predicted fragment ions are also detailed above (left) of the spectrum.

nated end groups, which have been shown to contain some peaks originating from loss of parts of this end group and the polymer'backbone 911,916$]$, in addition'to the expected fragmentation for this methacrylate. The data from this table can be exported from the software, as a comma separated value (.csv) file to be used in other applications.

Good agreement is shown between the theoretical and experimental mass-to-charge ratios, using the proposed fragment ion schemes that result in seven major series $^{\circ}$ of ${ }^{\circ}$ fragment ${ }^{\circ}$ ions ${ }^{\circ}\left[7,{ }^{\circ} 8,{ }^{\circ} 14\right] .{ }^{\circ}{ }^{\circ}$ The $^{\circ}$ series $^{\circ}$ are ${ }^{\circ}$ annotated by the letters $\mathrm{A} n, \mathrm{~B} n, \mathrm{C} n, \mathrm{D} n, \mathrm{E} n, \mathrm{~F} n$, and $\mathrm{G} n$, where $n$ is the number of repeat units of the monomer (methyl methacrylate) remaining in the fragment ions, as described in this previous work. Peaks from the An, $\mathrm{B} n$, and $\mathrm{G} n$ series are clearly annotated in the full spectrum $^{\circ}$ in ${ }^{\circ}$ Figure $^{\circ} 1 .^{\circ}$ Peaks $^{\circ}$ from $^{\circ}$ series $^{\circ}$ of $^{\circ}$ minor intensity (i.e., $\mathrm{C} n, \mathrm{D} n, \mathrm{E} n$, and $\mathrm{F} n$ series) are shown in the screenshot of the expansion of the MS/MS spectrum from ${ }^{\circ}$ MMA, ${ }^{\circ}$ which ${ }^{\circ}{ }^{\circ}$ Figure $2 .{ }^{\circ}$ Peaks ${ }^{\circ}$ from ${ }^{\circ}$ these ${ }^{\circ}$ series are typically observed at relative intensity values of low or subpercentage, compared to the intensities of peaks from ${ }^{\circ}$ the ${ }^{\circ} \mathrm{A} n$ and ${ }^{\circ} \mathrm{B} n$ series $^{\circ}\left[7,{ }^{\circ} 14\right] .{ }^{\circ}$ Peaks ${ }^{\circ}$ from $^{\circ}$ the ${ }^{\circ} \mathrm{G} n$ series are isobaric with those from the $\mathrm{C} n$ series-thus the labeling of peaks with annotation for both sets of fragment ions. Previous data indicated that the $G n$ series is dominant at lower mass-to-charge ratios, with the $\mathrm{C} n$ series becoming more intense at higher values. This explains the ratios of peaks from this series compared to those from the $\mathrm{D} n, \mathrm{E} n$, and $\mathrm{F} n$ series. The software is clearly capable of annotating the monoisotopic peaks from all of these series, even though they are of low relative intensity. An increase in the value (from ${ }^{\circ} 0.2^{\circ}$ to $^{\circ} 1.5^{\circ} \mathrm{Da}^{\circ}{ }^{\circ}{ }^{\circ}$ Figure $\left.^{\circ} 2\right)^{\circ}$ entered $^{\circ}$ into ${ }^{\circ}$ the ${ }^{\circ /}$ Error $(\mathrm{Da})^{\prime \prime}$ box can, additionally, enable labeling of the second peak from the isotope cluster of any or all fragment ions for this spectrum (depending on whether the value is entered for an individual peak or the whole spectrum). Both of the major peaks from the isotope cluster would, therefore, be annotated in this example.

Another example of how the software can be used to aid interpretation of MS/MS data from another methacrylate [poly(butyl methacrylate), 2] polymer has also been generated (data not shown). The same series of fragment ions were noted for the undecamer of poly(butyl methacrylate), as expected from previous work on this ${ }^{\circ}$ polymer [8]. The ${ }^{\circ}$ software ${ }^{\circ}{ }^{\circ}{ }^{\circ}$ also ${ }^{\circ}$ been ${ }^{\circ}$ successfully used to annotate MS/MS spectra from other classes of methacrylate polymer (data not shown), using the previously proposed fragmentation pathways.

Another polymer that has been extensively studied by ${ }^{\circ} \mathrm{MS} / \mathrm{MS}^{\circ}{ }^{\circ} \mathrm{s}^{\circ}$ poly(styrene) $)^{\circ}\left[9,{ }^{\circ} 10,{ }^{\circ} 12\right] .{ }^{\circ}$ Polymers $^{\circ}$ with $^{\circ} \mathrm{a}$ 


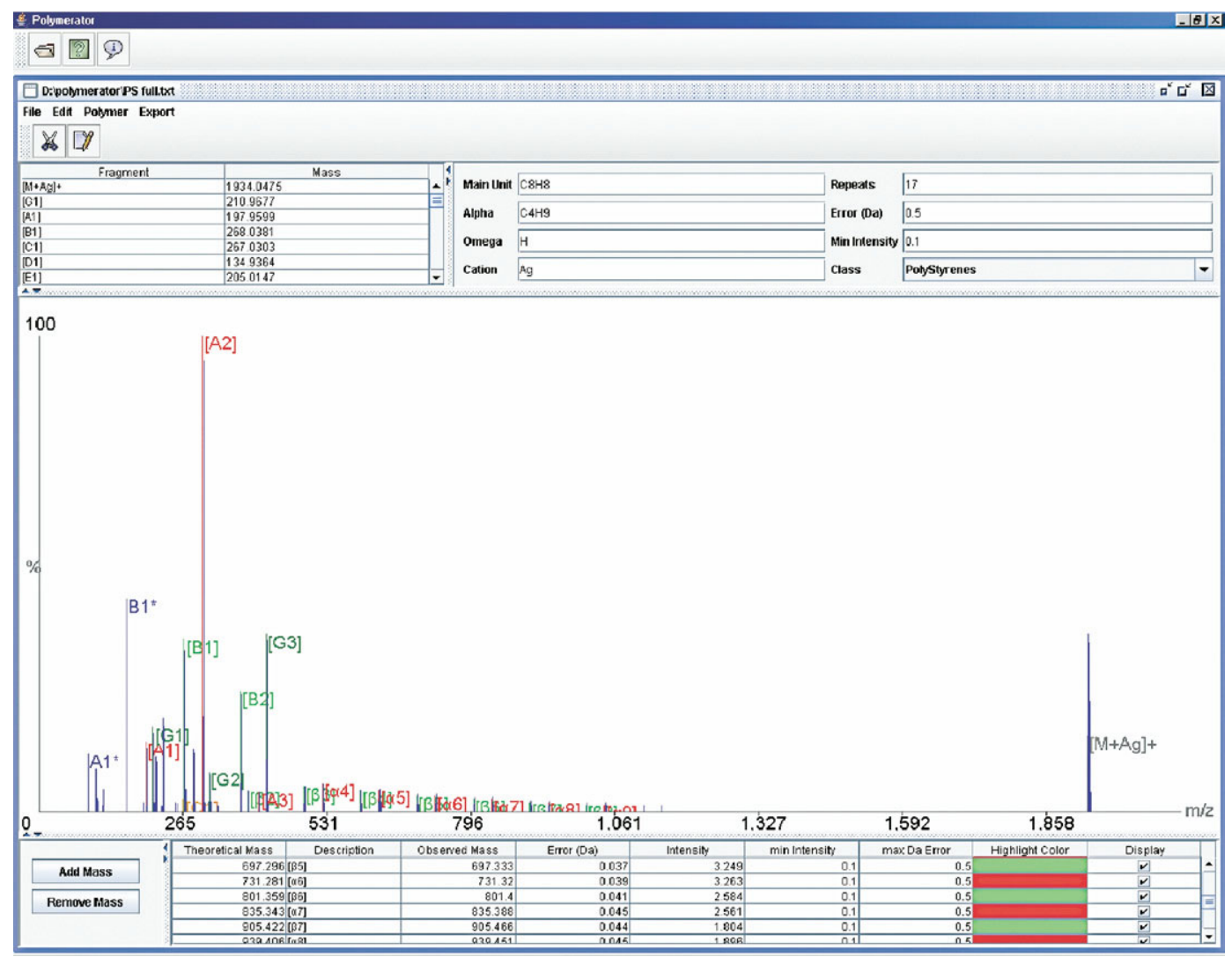

Figure 3. Screenshot from software of annotated ESI-MS/MS spectrum from the $[\mathrm{M}+\mathrm{Ag}]^{+}$adduct of the heptadecamer of poly(styrene). Details of annotated fragment ions are displayed by the software in the table below the spectrum. Predicted fragment ions are also detailed above (left) of the spectrum.

range of end-group functionalities, arising from variation in both initiators and end-capping agents, were clearly differentiated by the intense series of peaks (at low mass-to-charge ratios) noted in the MS/MS spectra [12]..$^{\circ}$ The $^{\circ}$ centroided $^{\circ}$ ESI-MS $/$ MS $^{\circ}$ spectrum $^{\circ}$ from $^{\circ}$ the silver cationized $\left([\mathrm{M}+\mathrm{Ag}]^{+}\right)$of the heptadecamer of poly(styrene) (3) was input into the software and the proposed empirical formulas of the initiating and terminating end groups and repeat unit were entered ( $\mathrm{C} 4 \mathrm{H} 9, \mathrm{H}$ and $\mathrm{C} 8 \mathrm{H} 8$, respectively). The cation $(\mathrm{Ag})$, number ${ }^{\circ}$ of $^{\circ}$ repeat $^{\circ}$ units $^{\circ}[17],{ }^{\circ}$ the $^{\circ}$ expected $^{\circ}$ maximum error in $m / z$ [0.5 in the "Error (Da)" input box] and minimum intensity to be annotated ( 0.1 in "Min Intensity" input box). The class of polymer chosen was "PolyStyrenes" before annotation of the MS/MS spectrum, ${ }^{\circ}$ resulting ${ }^{\circ}$ in $^{\circ}$ the $^{\circ}$ screenshot $^{\circ}$ displayed $^{\circ}{ }^{\circ}{ }^{\circ}$ Figure $^{\circ} 3$. The major series of peaks expected in the spectrum were annotated in milliseconds. The monoisotopic peaks from $\mathrm{A} n, \mathrm{~B} n, \alpha n, \beta n$, and $\mathrm{G} n$ series are clearly annotated in the spectrum displayed in the software, where $n$ is the number of repeat units of the monomer (styrene) remaining in the fragment ions. This annotation is similar'to that ${ }^{\circ}$ sed in ${ }^{\circ}$ arlier ${ }^{9}$ work $[9,910,12]$. The $\mathrm{A} n, \mathrm{~B} n$, and $\mathrm{G} n$ series are analogous to those noted for methacrylate ${ }^{\circ}$ polymers ${ }^{\circ}[8,99],{ }^{\circ}$ with ${ }^{\circ}$ the $^{\circ} \alpha n$ and $^{\circ} \beta n$ series seen only in spectra from styrenes. The former two series ( $\mathrm{A} n$ and $\mathrm{B} n)$ were previously proposed to be useful $^{\circ}$ for $^{\circ}$ end-group $^{\circ}$ identification $^{\circ}$ of $^{\circ}$ styrenes ${ }^{\circ}\left[9,{ }^{\circ} 10\right.$, 12]. The ${ }^{\circ}$ precursorion ${ }^{\circ}$ selection ${ }^{\circ}$ used in ${ }^{\circ}$ the ${ }^{\circ} \mathrm{ESI}-\mathrm{MS} / \mathrm{MS}$ experiment allowed both ions containing both ${ }^{107} \mathrm{Ag}$ and ${ }^{109} \mathrm{Ag}$ isotopes into the hexapole collision cell of the instrument, with peaks from fragment ions containing both moieties clearly observed in the spectra. The "Error (Da)" value (0.5) selected in the software meant that only the fragments containing ${ }^{107} \mathrm{Ag}$ were annotated, ${ }^{\circ}$ as $^{\circ} \operatorname{can}^{\circ}$ be $^{\circ}$ noted ${ }^{\circ}$ in $^{\circ}$ Figure $^{\circ} 3$.

In addition to the fragment ions containing silver isotopes (noted by the separation of $\mathrm{m} / \mathrm{z} 2$ between peaks of similar intensity for ions containing ${ }^{107} \mathrm{Ag}$ and ${ }^{109} \mathrm{Ag}$ ), peaks that resulted from ions that clearly did not contain silver were also observed at $\mathrm{m} / \mathrm{z} 91$ and 161 . These peaks were previously ascribed to fragments of the $\mathrm{A} n$ and $\mathrm{B} n$ series, respectively, that did not contain the $^{\circ}$ silver $^{\circ}$ cation $^{\circ}\left[9,^{\circ} 10,{ }^{\circ} 12\right] .^{\circ}$ These $^{\circ}$ peaks $^{\circ}$ have $^{\circ}$ been manually annotated with the software (see $\mathrm{A} 1^{*}$ and $\mathrm{B} 1^{*}$ in ${ }^{\circ}$ Figure $\left.{ }^{\circ}\right)^{\circ}$ using $^{\circ}$ the ${ }^{\text {or }}$ Add ${ }^{\circ}$ Mass" ${ }^{\circ}$ tool. ${ }^{\circ}$ The ${ }^{\circ}$ theoretical mass can be entered into the table for each additional peak to be entered, with custom annotation also added by the "Description" column of the table in the software. This flexibility allows custom annotation for any fragmentation not yet described in the literature. Custom annotation for peaks already captured by the 


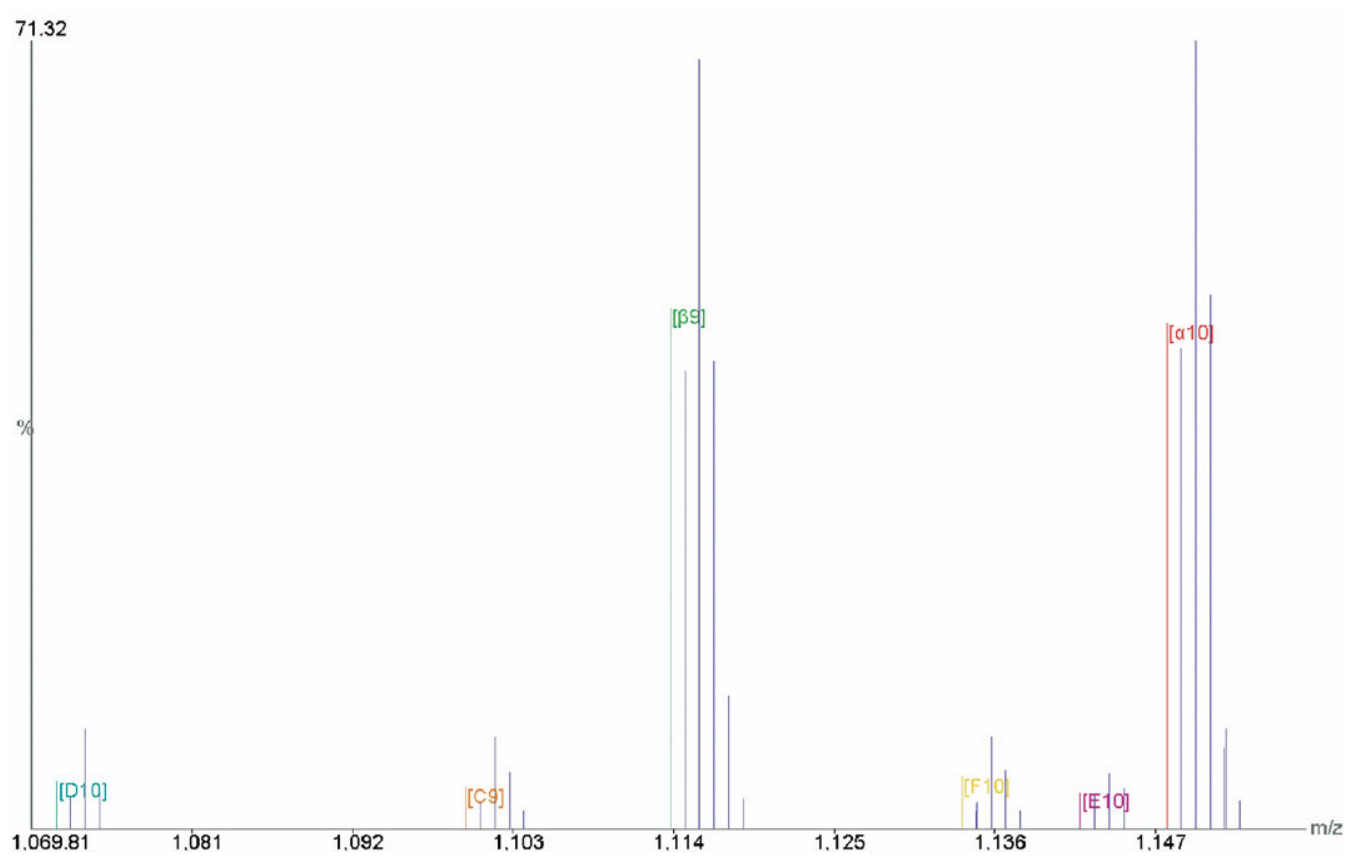

Figure 4. Screenshot from software of annotated expansion $(\mathrm{m} / \mathrm{z} \cong 1070-1160)$ of ESI-MS/MS spectrum from the $[\mathrm{M}+\mathrm{Ag}]^{+}$adduct of the heptadecamer of poly(styrene).

software is also possible by changing the entry for that moiety in the "Description" column of the table.

Annotation of some of the minor series noted to be present in MS/MS spectra from poly(styrene) can be noted ${ }^{9}$ ' Figure 4 , ' which $1 s^{\circ}$ a screenshot ${ }^{\circ}$ of ${ }^{\text {the }}{ }^{\circ}$ expanded spectrum from the heptadecamer. Peaks of very low relative intensity (again containing ${ }^{107} \mathrm{Ag}$ isotopes) are clearly annotated (from the $\mathrm{C} n, \mathrm{D} n, \mathrm{E} n$, and $\mathrm{F} n$ series) along with those from more abundant ions $(\beta 9$ and $\alpha 10)$. The $\mathrm{C} n, \mathrm{D} n, \mathrm{E} n$, and $\mathrm{F} n$ series are typically noted to be present at low relative intensities in MS/MS spectra from poly(styrene)s. An additional example of the software for $\operatorname{poly}(\alpha$-methyl styrene) (4) was used to indicate the application for other styrene based polymers (data not shown). Similar series of fragment ions were noted in the annotated spectrum of the $[\mathrm{M}+\mathrm{Ag}]^{+}$ adduct of the heptamer of poly( $\alpha$-methyl styrene) as described ${ }^{\circ}$ previously ${ }^{\circ}[22]$.

Lattimer initially demonstrated that MS/MS could be used to generate structural information from 1,2epoxide derived polymers such as poly(ethylene glycol) (PEG) ${ }^{\circ}$ and ${ }^{\circ}$ poly (propylene ${ }^{\circ}$ glycol) ${ }^{\circ}(\mathrm{PPG}){ }^{\circ}[19,20]$. Mechanisms were proposed to account for the fragment ions generated when lithium was used as the cationization agent. Three dominant series were noted to be present in the spectra. A range of other polyethers have more recently ${ }^{\circ}$ been $^{\circ}{ }^{\circ}$ characterized ${ }^{\circ}\left[15,{ }^{\circ} 17\right],{ }^{\circ}$ with ${ }^{\circ}$ similar $^{\circ}$ fragmentation noted to dominate in most cases, with the

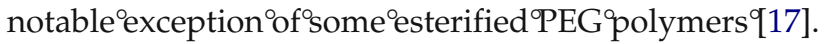
The centroided ESI-MS/MS spectrum from the lithiated decamer of PEG (5) was imported into the software as a text file and the peaks annotated after entering values for the alpha $(\mathrm{H})$ and omega $(\mathrm{H})$ end groups, repeat unit
(C2H4O), number of repeats (10), cation ( $\mathrm{Li})$, minimum relative intensity for fragments to be annotated (1), and the mass accuracy [0.04 in "Error (Da)"]. The selected polymer class was "Polyethers," which encompasses polymers such as PEG, PPG, and poly(butylene glycol). The ${ }^{\circ}$ resulting ${ }^{\circ}$ screenshot ${ }^{\circ}{ }^{\circ}{ }^{\circ}$ displayed ${ }^{\circ}$ in ${ }^{\circ}$ Figure $^{\circ} 5,{ }^{\circ}$ with fragment and precursor $\left([\mathrm{M}+\mathrm{Li}]^{+}\right)$peaks annotated.

Peaks from the $\mathrm{A} n, \mathrm{~B} n, \mathrm{C} n$, and $\mathrm{D} n$ series are annotated, corresponding to that originally proposed by $^{\circ}$ Lattimer $^{\circ}\left[19,{ }^{\circ} 20\right]^{\circ}$ and $^{\circ}$ recently $^{\circ}$ modified $^{\circ}[17]^{\circ}$ to account for the number of repeat units $(n)$ remaining in the fragment ions. The $\mathrm{A} n, \mathrm{~B} n$, and $\mathrm{C} n$ series were proposed to be key in generating information on structure $^{\circ}$ of $^{\circ}$ the ${ }^{\circ}$ end $^{\circ}$ groups $^{\circ}[17],{ }^{\circ}$ with $^{\circ}$ the $e^{\circ}$ higher ${ }^{\circ} \mathrm{m} / z$ fragment ions being the most informative. The mass accuracy of the ESI-MS/MS experiment, with the precursor ion used as a lock mass, enabled further confirmation of the structures of the fragment ions and thus the end group structure. The capability to use nominal or exactmass data is another key feature of the software. The Dn series $^{\circ}$ were $^{\circ}$ proposed $^{\circ}$ to $^{\circ}$ be $^{\circ}$ distonic $^{\circ}$ radical $^{\circ}$ cations $^{\circ}[19$, 20], ${ }^{\circ}$ typically ${ }^{\circ}$ observed $^{\circ}{ }^{\circ} t^{\circ}{ }^{\circ}$ ow $^{\circ}$ mass-to-charge ${ }^{\circ}$ ratios $^{\circ}$ as noted in the screenshot ${ }^{\circ}$ hown in ${ }^{\circ}$ Figure 5 . The software differentiates between fragment ions resulting from retention of either end group, when these functionalities are different. The fragment ion series containing the initiating group are clearly differentiated by extra annotation from those containing the terminating moiety, 'using ${ }^{\circ}$ the ${ }^{\circ}$ recently ${ }^{\circ}$ proposed ${ }^{\text {nnomenclature }}[17]^{\text {th }}$ that was modified from that originally described by Lattimer $[19,20]^{\circ}$ [because $^{\circ}$ the ${ }^{\circ}$ end $^{\circ}$ groups $^{\circ}{ }^{\circ}{ }^{\circ}{ }^{\circ}$ equivalent ${ }^{\circ}$ in this ${ }^{\circ}$ example $^{\circ}$ (Figure ${ }^{\circ}$ ), ${ }^{\circ}$ the ${ }^{\circ}$ fragment ${ }^{\circ}$ series ${ }^{\circ}$ are ${ }^{\circ}$ labeled without this extra annotation by the software]. 


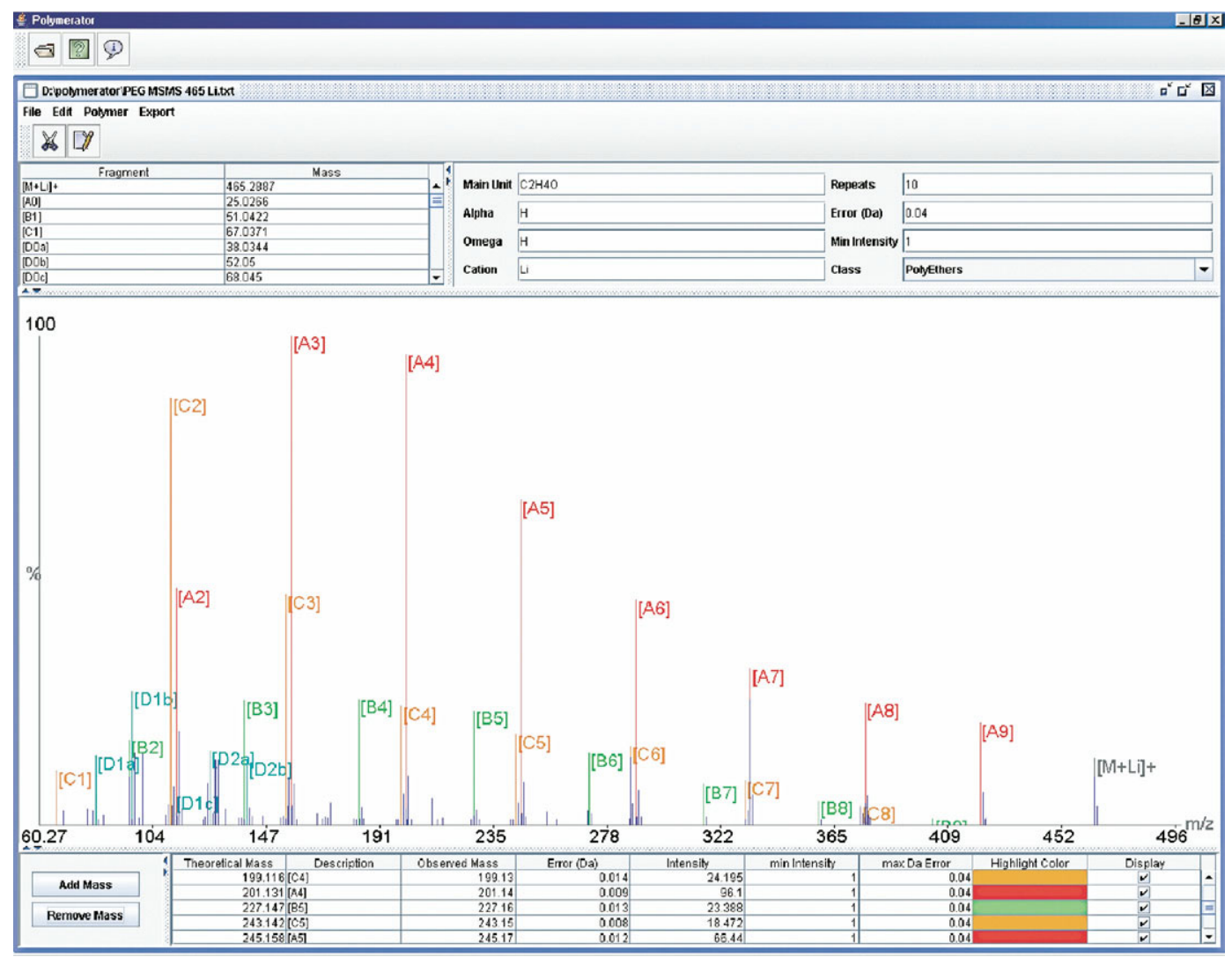

Figure 5. Screenshot from software of annotated ESI-MS/MS spectrum from the lithiated decamer of PEG. Details of annotated fragment ions are displayed by the software in the table below the spectrum. Predicted fragment ions are also detailed above (left) of the spectrum.

An example for PPG (6) was also generated to show the application of the software for other polyethers (data not shown). Similar fragmentation pathways were noted, from the lithiated heptamer of PPG, to that expected ${ }^{\circ}$ from ${ }^{\circ}$ previous $^{\circ}$ work $^{\circ}[17,19,22]$. The ${ }^{\circ}$ software can also be used to predict a fragment ion spectrum for a particular structure by entering the relevant values into the input boxes and predicting the spectrum. The proposed fragment ion mass-to-charge ratios and annotation are then displayed in the table to the left of the input boxes. These data can then be exported as a comma separated value (.csv) file to be used elsewhere.

\section{Conclusions}

The software aids the interpretation of MS/MS spectra from polymers with known fragmentation pathways, such as methacrylates, polyethers, and polystyrenes, but the capability to interpret other polymer systems can easily be added to improve the applicability to these additional materials. Rapid annotation of the MS/MS data, using the software, significantly reduces the time taken for interpretation (compared to manual annotation, which can be difficult and/or laborious). The software has the capability to use exact-mass MS/MS data for further confidence in the results. The software enables novel structural features to be more easily identified, by characterizing peaks from all known fragmentation pathways.

The software is platform independent and can be run in any operating system having the Java runtime installed. It is also proprietary vendor format independent because the input for the software is a text file containing fragment mass intensity information. Theoretical fragment generation and peak annotation are instantaneous. Annotation information (images, text files) can be printed and/or exported for use in other programs. The entire process requires minimal intervention from users. Developments of the approach to enable automatic identification of end groups and studies of random and block copolymers are under way.

\section{Acknowledgments}

The authors gratefully acknowledge the contributions of the Biological Mass Spectrometry and Proteomics Facility in the Department of Biological Sciences, University of Warwick.

\section{References}

1. Eng, J. K.; McCormack, A. L.; Yates, J. R. An Approach to Correlate Tandem Mass Spectra Data of Peptides with Amino Acid Sequences in a Protein Database. J. Am. Soc. Mass Spectrom. 1994, 5, 976-989.

2. Perkins, D. N.; Pappin, D. J.; Creasy, D. M.; Cottrell, J. S. ProbabilityBased Protein Identification by Searching Sequence Databases Using Mass Spectrometry Data. Electrophoresis 1999, 20, 3551-3567. 
3. Zhang, N.; Aebersold, R.; Schwikowski, B. ProbID: A Probabilistic Algorithm to Identify Peptides through Sequence Database Searching Using Tandem Mass Spectral Data. Proteomics 2002, 2, 1406-1412.

4. Craig, R.; Beavis, R. C. A Method for Reducing the Time Required to Match Protein Sequences with Tandem Mass Spectra. Rapid Commun. Mass Spectrom. 2003, 17, 2310-2316.

5. Ma, B.; Zhang, K.; Hendrie, C.; Liang, C.; Li, M.; Doherty-Kirby, A.; Lajoie, G. PEAKS: Powerful Software for Peptide De Novo Sequencing by Tandem Mass Spectrometry. Rapid Commun. Mass Spectrom. 2003, 17, 2337-2342.

6. Geer, L. Y.; Markey, S. P.; Kowalak, J. A.; Wagner, L.; Xu, M.; Maynard, D. M.; Yang, X.; Shi, W.; Bryant, S. H. Open Mass Spectrometry Search Algorithm. J. Proteome Res. 2004, 3, 958-964.

7. Jackson, A. T.; Yates, H. T.; Scrivens, J. H.; Critchley, G.; Brown, J.; Green, M. R.; Bateman, R. H. The Application of Matrix-Assisted Laser Desorption/Ionization Combined with Collision-Induced Dissociation to the Analysis of Synthetic Polymers. Rapid Commun. Mass Spectrom. 1996, 10, 1668-1674.

8. Jackson, A. T.; Yates, H. T.; Scrivens, J. H.; Green, M. R.; Bateman, R. H. Utilizing Matrix-Assisted Laser Desorption/Ionization-Collision Induced Dissociation for the Generation of Structural Information from Poly(alkyl Methacrylate)s. J. Am. Soc. Mass Spectrom. 1997, 8, 1206-1213.

9. Scrivens, J. H.; Jackson, A. T.; Yates, H. T.; Green, M. R.; Critchley, G.; Brown, J.; Bateman, R. H.; Bowers, M. T.; Gidden, J. The Effect of the Variation of Cation in the Matrix-Assisted Laser Desorption/Ionisation Collision Induced Dissociation (MALDI-CID) Spectra of Oligomeric Systems. Int. J. Mass Spectrom. 1997, 165, 363-375.

10. Jackson, A. T.; Yates, H. T.; Scrivens, J. H.; Green, M. R.; Bateman, R. H. Matrix-Assisted Laser Desorption/Ionization-Collision Induced Dissociation of Poly(styrene). J. Am. Soc. Mass Spectrom. 1998, 9, 269-274.

11. Borman, C. D.; Jackson, A. T.; Bunn, A.; Cutter, A. L.; Irvine, D. J. Evidence for the Low Thermal Stability of Poly(methyl methacrylate) Polymer Produced by Atom Transfer Radical Polymerisation. Polymer 2000, 41, 6015-6020.

12. Jackson, A. T.; Bunn, A.; Hutchings, L. R.; Kiff, F. T.; Richards, R. W.; Williams, J.; Green, M. R.; Bateman, R. H. The Generation of End Group
Information from Poly(styrene)s by Means of Matrix-Assisted Laser Desorption/Ionisation-Collision Induced Dissociation. Polymer 2000, 41 7437-7450.

13. Jackson, A. T.; Scrivens, J. H.; Simonsick, W. J.; Green, M. R.; Bateman R. H. Generation of Structural Information from Polymers and Copolymers Using Tandem Mass Spectrometry. Abstr. Pap. Am. Chem. Soc. 2000, 219, U363.

14. Jackson, A. T.; Slade, S. E.; Scrivens, J. H. Characterisation of Poly(alkyl methacrylate)s by Means of Electrospray Ionisation-Tandem Mass Spectrometry (ESI-MS/MS). Int. J. Mass Spectrom. 2004, 238, 265-277.

15. Jackson, A. T.; Scrivens, J. H.; Williams, J. P.; Baker, E. S.; Gidden, J. Bowers, M. T. Microstructural and Conformational Studies of Polyether Copolymers. Int. J. Mass Spectrom. 2004, 238, 287-297.

16. Jackson, A. T.; Bunn, A.; Priestnall, I. M.; Borman, C. D.; Irvine, D. J. Molecular Spectroscopic Characterisation of Poly(methyl methacrylate) Generated by Means of Atom Transfer Radical Polymerisation (ATRP). Polymer 2006, 47, 1044-1054.

17. Jackson, A. T.; Green, M. R.; Bateman, R. H. Generation of End-Group Information from Polyethers by Matrix-Assisted Laser Desorption/ Ionisation Collision-Induced Dissociation Mass Spectrometry. Rapid Commun. Mass Spectrom. 2006, 20, 3542-3550.

18. Lattimer, R. P. Tandem Mass-Spectrometry of Poly(Ethylene Glycol) Proton-Attachment and Deuteron-Attachment Ions. Int. J. Mass Spectrom. Ion. Process. 1992, 116, 23-36.

19. Lattimer, R. P. Tandem Mass-Spectrometry of Lithium-Attachment Ions from Polyglycols. J. Am. Soc. Mass Spectrom. 1992, 3, 225-234.

20. Lattimer, R. P. Tandem Mass-Spectrometry of Poly(Ethylene Glycol) Lithium-Attachment Ions. J. Am. Soc. Mass Spectrom. 1994, 5, 1072 1080 .

21. Selby, T. L.; Wesdemiotis, C.; Lattimer, R. P. Dissociation Characteristics of $[\mathrm{M}+\mathrm{X}](+)$ Ions $(\mathrm{X}=\mathrm{H}, \mathrm{Li}, \mathrm{K})$ from Linear and Cyclic Polyglycols. J. Am. Soc. Mass Spectrom. 1994, 5, 1081-1092.

22. Jackson, A. T.; Williams, J. P.; Scrivens, J. H. Desorption Electrospray Ionisation Mass Spectrometry and Tandem Mass Spectrometry of Low Molecular Weight Synthetic Polymers. Rapid Commun. Mass Spectrom. 2006, 20, 2717-2727. 